\title{
A simple approach to multiple attribute decision making using loss functions
}

\author{
Vijaya Babu Vommi ${ }^{1}$ - Sravya Roy Kakollu ${ }^{1}$
}

Received: 10 March 2016/ Accepted: 30 September 2016/Published online: 15 October 2016

(c) The Author(s) 2016. This article is published with open access at Springerlink.com

\begin{abstract}
Multiple attribute decision making (MADM) methods are very much essential in all fields of engineering, management and other areas where limited alternatives exist and the decision maker has to select the best alternative. Different methods are available in the literature to tackle the MADM problems. The MADM problems are classified as scoring methods, compromising methods and concordance methods. The concordance methods are difficult to understand compared to scoring and compromising methods. Present work introduces a simple-to-understand and easy-to-convince method for multiple attribute decision making problems. This method is based on the philosophy of both scoring and comprising methods and relies on the loss for not choosing the ideal best alternative. Different loss functions such as linear, quadratic and cubic functions have been proposed to calculate the loss. Example problems have been taken from the literature and the proposed method is implemented. Besides the simplicity of the proposed method, the results obtained are found to be in close agreement with rather difficult methods.
\end{abstract}

Keywords Multiple attribute decision making - Ideal best alternative $\cdot$ Loss functions

Vijaya Babu Vommi

vvijayababu.mech@auvsp.edu.in

1 Department of Mechanical Engineering, Andhra University, Visakhapatnam 530 003, India

\section{Introduction}

Multiple criteria decision making (MCDM) problems are studied mainly under two categories, namely multiple objective decision making (MODM) problems and multiple attribute decision making (MADM) problems. The MODM problems emphasize the design of best alternative wherein the alternatives are not predetermined. In contrast, the number of alternatives in MADM problems is predetermined and is usually limited. Hence, it can be stated that MODM problems are concerned with design whereas the MADM problems are used for selection (Hwang and Yoon 1981). The MADM methods are classified as non-compensatory and compensatory. Some of the non-compensatory methods include maxmin, maximax, dominance, conjunctive constraint method, and lexicographic method. These methods are simple but their applications are limited. The compensatory models are very popular. These methods can be described under three categories: (1) scoring methods (2) compromising methods and (3) concordance methods (Hwang and Yoon 1981). A taxonomy of methods for classical MADM problems and fuzzy ranking methods can be found in the study by Chen and Hwang (1992).

Scoring methods prefer the alternative which has the highest score. The methods based on MAUT (Multi-attribute utility theory; Keeney and Raffia 1993) belong to this category. Simple additive weighting (SAW) summarized by McCrimmon (1968) and analytical hierarchy process (AHP) developed by Saaty (1980) are very popular scoring methods. The compromising methods rely on the ideal best and ideal worst solutions obtained from the available alternatives. TOPSIS (The Technique for Order of Preference by Similarity to Ideal Solution) developed by Hwang and Yoon (1981) and VIKOR (VIsekriterijumsko 
KOmpromisno Rangiranje) developed by Zeleny (1982) are very popular in this category. Some new approaches based on the above scoring and compromising methods can be found in the studies by Yang et al. (2013), Wang et al. (2015) and Qin et al. (2015). The ELECTRE (Elimination et Choice Translating Reality) method introduced by Benayoun et al (1966) and the PROMETHEE (Preference Ranking Organization Method for Enrichment Evaluation) method introduced by Brans et al. (1984) are very popular concordance methods.

Many other techniques like COPRAS (Complex proportional assessment; Zavadskas et al. 2008), DEA (Data Envelopment Analysis; Charnes et al. 1978), DEMATEL (Decision making trial and evaluation laboratory; Fontela and Gabus 1974, 1976), GTMA (Graph theory and matrix analysis; Rao 2007, 2013) GRA (Gray relational analysis; Deng 1989), MACBETH (Measuring attractiveness by a categorical-based evaluation technique; Bana e Costa and Vansnick 1997), MOORA (Multi-objective optimization on the basis of ratio analysis; Brauers and Zavadskas 2006), MULTIMOORA - a comprehensive extension of MOORA (Brauers and Zavadskas 2010; Hafezalkotob and Hafezalkotob 2016), OWA(Ordered weighted averaging; Yager 1988), RST (Rough set theory; Pawlak and Slowinski 1994), and UTA (Utilities additives; JacquetLagreze and Siskos 1982) are also employed for solving the MADM problems.

In the present work, a simple method for MADM problems has been introduced based on loss functions. This method uses the philosophy of both scoring and compromising methods. The paper is organized as follows: in the next section, the proposed loss function approach is described and the total loss for choosing an alternative is derived. The subsequent section deals with the application of the proposed loss function approach to decision making problems taken from the literature and comparison of the results with other popular methods. Summary and concluding remarks are given in the final section.

\section{The loss function approach and total loss derivation}

In the present paper, a simple and convincing method to MADM has been proposed. The present method relies on the principle of measuring the loss caused by each attribute for not being the best with respect to the best value available among all the alternatives.

Multiple attribute decision making problems pose a challenge to the decision maker to select the best alternative from among the set of alternatives. Each alternative consists of a few attributes based on which the decision maker chooses the best alternative. The attributes are in general of two types, namely beneficial and non-beneficial. In the case of beneficial attributes, higher values are desired and for non-beneficial attributes lower values are preferred. In general, no alternative will possess all beneficial attributes at higher values and all non-beneficial attributes at lower values. Some alternatives may possess best values with respect to some attributes and may possess undesired values at higher levels with respect to the other. In addition, the attributes will possess different weights with respect to each other. Because of this conflicting nature, the decision maker has to choose the best alternative based on some criterion. For example, in TOPSIS methodology, the best alternative is chosen based on the Euclidean distances of the alternatives from the ideal best and ideal worst alternatives. Irrespective of the nature of attribute, in TOPSIS methodology, the Euclidean distances only will be considered. But, in reality, the effect of the attribute need not always be proportional to the straight line distance only. For example, in Taguchi's loss function approach (Kackar 1985), the losses are taken proportional to the square of the deviation of the quality characteristic from the desired nominal value.

In the proposed method, initially, the ideal best alternative has to be obtained from the available alternatives. The ideal best alternative consists of all higher values for the beneficial attributes and all lower values for the nonbeneficial values. The loss for choosing each alternative has to be calculated with respect to the ideal best alternative. The alternative with lowest possible total loss is chosen as the best alternative. The losses can be calculated not only based on quadratic function, but they can be calculated using linear and cubic loss functions also.

\section{Loss function approach (LFA)}

The decision matrix of an MADM problem with $n$ alternatives and $p$ attributes is shown in Table 1 . Let $w_{j}$ be the weight of the $j$ th attribute.

Let $x_{\mathrm{ij}}$ represent the value of $j$ th attribute in the $i$ th alternative.

The ideal best alternative (IBA) selected from data matrix of Table 1 can be represented mathematically as:

Table 1 Typical decision matrix

\begin{tabular}{llllllll}
\hline Alternatives & \multicolumn{3}{l}{ Attributes } & & & \\
\cline { 2 - 7 } & 1 & 2 & 3 & 4 & $\ldots \ldots \ldots \ldots \ldots \ldots \ldots \ldots \ldots$ & $p$ \\
\hline 1 & $x_{11}$ & $x_{12}$ & $x_{13}$ & $x_{14}$ & $\ldots \ldots \ldots \ldots \ldots \ldots \ldots \ldots \ldots$ & $x_{1 p}$ \\
2 & $x_{21}$ & $x_{22}$ & $x_{23}$ & $x_{24}$ & $\ldots \ldots \ldots \ldots \ldots \ldots \ldots \ldots \ldots \ldots$ & \\
$\ldots$ & $\ldots$. & $\ldots \ldots$ & $\ldots \ldots$ & $\ldots \ldots$ & $\ldots \ldots \ldots \ldots \ldots \ldots \ldots \ldots \ldots$ & \\
$n$ & $x_{n 1}$ & $x_{n 2}$ & $x_{n 3}$ & $x_{n 4}$ & $\ldots \ldots \ldots \ldots \ldots \ldots \ldots \ldots \ldots$ & $x_{n p}$
\end{tabular}




$$
\begin{gathered}
I B A=\left\{\max _{i}\left(x_{\mathrm{ij}} \mid j \varepsilon H\right), \min _{i}\left(x_{\mathrm{ij}} \mid j \varepsilon S\right)\right\} \\
i=1,2, \ldots \ldots \ldots n
\end{gathered}
$$

where $H$ and $S$ represent the set of beneficial attributes and non-beneficial attributes, respectively.

The attributes of any chosen alternative $(i=1,2$, ...n) have to be compared with the attributes of IBA and the loss for not being the best for each attribute has to be calculated. The sum of the losses caused by all attributes of an alternative gives the total loss for choosing a particular alternative.

\section{Maximum and minimum losses for attributes}

To calculate the total loss of any alternative, initially the loss caused by each attribute of that particular alternative has to be calculated. To calculate the loss caused by each attribute, the attributes of the chosen alternative are to be compared with those of IBA.

For a beneficial attribute, the minimum loss is taken to be zero when the attribute possesses the maximum value with respect to all alternatives under consideration. The loss is taken to be maximum when a beneficial attribute is at minimum value. On a zero-one scale, the loss is considered to be equal to one when the attribute value is at minimum with respect to all alternatives under consideration.

Using the same logic for a non-beneficial attribute, the minimum loss is taken as zero when the attribute value is at minimum and the maximum loss is taken as one when the attribute possesses the maximum value.

Let $x_{\max }^{j}$ represent the maximum value of the $j$ th attribute among all alternatives,

i.e.,

$x_{\max }^{j}=\max \left\{x_{\mathrm{ij}}\right\} \quad \forall i=1,2, \ldots \ldots \ldots n$

Let $x_{\min }^{j}$ represent the minimum value of the $j$ th attribute among all alternatives,

i.e.,

$x_{\min }^{j}=\min \left\{x_{\mathrm{ij}}\right\} \quad \forall i=1,2 \ldots \ldots \ldots . n$

The maximum and minimum losses $L^{j}$ for a beneficial attribute can be expressed mathematically as:

$L^{j}=\left\{\begin{array}{ll}0 & \text { if } x_{\mathrm{ij}}=x_{\max }^{j} \\ 1 & \text { if } x_{\mathrm{ij}}=x_{\min }^{j}\end{array} \quad j \in H\right.$

Similarly, the maximum and minimum losses $L^{j}$ for a non-beneficial attribute can be expressed mathematically as:

$L^{j}=\left\{\begin{array}{ll}0 & \text { if } x_{\mathrm{ij}}=x_{\min }^{j} \\ 1 & \text { if } x_{\mathrm{ij}}=x_{\max }^{j}\end{array} \quad j \in S\right.$
From Eqs. (4) and (5), it can be observed that the maximum loss is considered equal to 1 when the beneficial attribute is at minimum and the non-beneficial attributes at maximum values. But, all the attributes are not equally weighted in MADM problems. Hence, the maximum losses cannot be considered to be equal to 1 for all attributes. Incorporating the weights of different attributes, the maximum loss for each attribute is obtained by multiplying the maximum loss (equal to one) by the respective weight of the attribute.

Considering the individual weights of all attributes, the maximum and minimum losses of beneficial attributes can now be expressed as:

$L^{j}=\left\{\begin{aligned} 0 & \text { if } x_{\mathrm{ij}}=x_{\max }^{j} \\ w_{\mathrm{H}}^{j} & \text { if } x_{\mathrm{ij}}=x_{\min }^{j}\end{aligned} \quad j \in H\right.$

Similarly, the maximum and minimum losses for a nonbeneficial attributes can be expressed as:

$L^{j}=\left\{\begin{array}{cl}0 & \text { if } x_{\mathrm{ij}}=x_{\min }^{j} \\ w_{\mathrm{S}}^{j} & \text { if } x_{\mathrm{ij}}=x_{\max }^{j}\end{array} \quad j \in S\right.$

where $w_{\mathrm{S}}^{j}$ and $w_{\mathrm{H}}^{j}$ represent the weights of non-beneficial and beneficial attributes, respectively.

\section{Loss calculation for a non-beneficial/beneficial attribute}

In this section, the loss equations for non-beneficial/beneficial attributes are derived for linear, quadratic and cubic functions. In case of linear function, the loss is assumed to be proportional to the deviation of the attribute from the best value of the attribute. Similarly, in quadratic loss function the loss is assumed to be proportional to the square of the deviation of the attribute from the best value of the attribute. Loss is assumed to be proportional to the cube of the deviations in case of cubic loss functions.

Assuming a linear loss function, i.e., the loss is proportional to the deviation, the loss function for the $j$ th attribute of any alternative can be written as:

$L\left(x_{\mathrm{ij}}\right)=K_{S}^{j}\left(x_{\mathrm{ij}}-x_{\min }^{j}\right)$

where $K_{\mathrm{S}}^{j}$ is the constant of proportionality for a non-beneficial attribute.

At

$x_{\mathrm{ij}}=x_{\min }^{j}$

$L\left(x_{\mathrm{ij}}\right)=0$.

At

$x_{\mathrm{ij}}=x_{\max }^{j}$

$L\left(x_{\mathrm{ij}}\right)=w_{\mathrm{S}}^{j}$.

Combining Eqs. (8) and (10), 
$w_{\mathrm{S}}^{j}=K_{\mathrm{S}}^{j}\left(x_{\max }^{j}-x_{\min }^{j}\right)$.

The constant of proportionality can be obtained from Eq. (11) as:

$K_{\mathrm{S}}^{j}=\frac{w_{\mathrm{s}}^{j}}{\left(x_{\max }^{j}-x_{\min }^{j}\right)}$.

Using the value of constant of proportionality from Eq. (12), the linear loss function for a non-beneficial attribute can be written as:

$L\left(x_{\mathrm{ij}}\right)=w_{\mathrm{S}}^{j}\left(x_{\mathrm{ij}}-x_{\min }^{j}\right) /\left(x_{\max }^{j}-x_{\min }^{j}\right)$.

Using quadratic loss function, the loss for a non-beneficial attribute can be calculated as:

$L\left(x_{\mathrm{ij}}\right)=K_{\mathrm{S}}^{j}\left(x_{\mathrm{ij}}-x_{\min }^{j}\right)^{2}$.

Substituting the conditions as in Eqs. (9) and (10), the constant of proportionality can be obtained as:

$K_{\mathrm{S}}^{j}=\frac{w_{\mathrm{s}}^{j}}{\left(x_{\max }^{j}-x_{\min }^{j}\right)^{2}}$.

Using Eqs. (14) and (15), the quadratic loss function for a non-beneficial attribute can be obtained as:

$L\left(x_{i j}\right)=w_{S}^{j}\left[\frac{\left(x_{i j}-x_{\min }^{j}\right)}{\left(x_{\max }^{j}-x_{\min }^{j}\right)}\right]^{2}$.

In the similar lines, the cubic loss function for a nonbeneficial attribute can be obtained as:

$L\left(x_{i \mathrm{j}}\right)=w_{\mathrm{S}}^{j}\left[\frac{\left(x_{\mathrm{ij}}-x_{\min }^{j}\right)}{\left(x_{\max }^{j}-x_{\min }^{j}\right)}\right]^{3}$.

Now, the linear loss function for a beneficial attribute can be obtained as:

$L\left(x_{\mathrm{ij}}\right)=w_{\mathrm{H}}^{j}\left(x_{\max }^{j}-x_{\mathrm{ij}}\right) /\left(x_{\max }^{j}-x_{\min }^{j}\right)$.

The quadratic loss function for a beneficial attribute can be obtained as:

$L\left(x_{\mathrm{ij}}\right)=w_{\mathrm{H}}^{j}\left[\frac{\left(x_{\max }^{j}-x_{i j}\right)}{\left(x_{\max }^{j}-x_{\min }^{j}\right)}\right]^{2}$.

The cubic loss function for beneficial attribute can be obtained as:

$L\left(x_{\mathrm{ij}}\right)=w_{\mathrm{H}}^{j}\left[\frac{\left(x_{\max }^{j}-x_{\mathrm{ij}}\right)}{\left(x_{\max }^{j}-x_{\min }^{j}\right)}\right]^{3}$.

Total loss calculation for an alternative

Assuming that any alternative in the decision matrix has a combination of beneficial and non-beneficial attributes, the total loss for choosing any alternative $i$ can be written as

$L_{\mathrm{i}}=L_{\mathrm{S}}+L_{\mathrm{H}}$

where $L_{\mathrm{H}}$ is the loss caused by the beneficial attributes and $L_{\mathrm{S}}$ is the loss caused by the non-beneficial attributes.

For a linear loss function, the loss caused by the nonbeneficial attributes of an alternative $i$ is given by:

$L_{\mathrm{S}}=\sum_{j \in S} \frac{w_{\mathrm{S}}^{j}\left(x_{\mathrm{ij}}-x_{\min }^{j}\right)}{\left(x_{\max }^{j}-x_{\min }^{j}\right)}$.

The loss caused by the beneficial attributes is given by:

$L_{\mathrm{H}}=\sum_{j \in H} \frac{w_{\mathrm{H}}^{j}\left(x_{\max }^{j}-x_{\mathrm{ij}}\right)}{\left(x_{\max }^{j}-x_{\min }^{j}\right)}$

The total loss caused by the alternative $i$ using linear loss function is given by:

$L_{\mathrm{i}}=\sum_{j \in S} \frac{w_{\mathrm{S}}^{j}\left(x_{\mathrm{ij}}-x_{\min }^{j}\right)}{\left(x_{\max }^{j}-x_{\min }^{j}\right)}+\sum_{j \varepsilon H} \frac{w_{\mathrm{H}}^{j}\left(x_{\max }^{j}-x_{\mathrm{ij}}\right)}{\left(x_{\max }^{j}-x_{\min }^{j}\right)}$.

The total loss caused by the alternative $i$ using quadratic loss function is given by:

$L_{\mathrm{i}}=\sum_{j \in S} \frac{w_{\mathrm{S}}^{j}\left(x_{\mathrm{ij}}-x_{\min }^{j}\right)^{2}}{\left(x_{\max }^{j}-x_{\min }^{j}\right)^{2}}+\sum_{j \varepsilon H} \frac{w_{\mathrm{H}}^{j}\left(x_{\max }^{j}-x_{\mathrm{ij}}\right)^{2}}{\left(x_{\max }^{j}-x_{\min }^{j}\right)^{2}}$.

The total loss caused by the alternative $i$ using cubic loss function is given by:

$L_{\mathrm{i}}=\sum_{j \in S} \frac{w_{\mathrm{S}}^{j}\left(x_{\mathrm{ij}}-x_{\min }^{j}\right)^{3}}{\left(x_{\max }^{j}-x_{\min }^{j}\right)^{3}}+\sum_{j \varepsilon H} \frac{w_{H}^{j}\left(x_{\max }^{j}-x_{\mathrm{ij}}\right)^{3}}{\left(x_{\max }^{j}-x_{\min }^{j}\right)^{3}}$.

In a general form, the total loss caused by the alternative $i$ can be written as:

$L_{\mathrm{i}}=\sum_{j \in S} \frac{w_{\mathrm{S}}^{j}\left(x_{\mathrm{ij}}-x_{\min }^{j}\right)^{n}}{\left(x_{\max }^{j}-x_{\min }^{j}\right)^{n}}+\sum_{j \varepsilon H} \frac{w_{H}^{j}\left(x_{\max }^{j}-x_{\mathrm{ij}}\right)^{n}}{\left(x_{\max }^{j}-x_{\min }^{j}\right)^{n}}$

where $n$ is the index of loss function. For $n=1,2$ and 3, the loss function is linear, quadratic and cubic, respectively. The loss function approach with loss function index $n$ is designated as LFA- $n$.

\section{Background and discussion on the loss function approach}

Use of the city block distance as a separation measure can be found in the technique SMART (Similarity Measure Anchored Ranking Technique) introduced by Dasarathy (1976). The TOPSIS technique introduced by Hwang and Yoon (1981) is simple in its logic and relies on the Euclidean distances of the alternative under consideration 
from the ideal best and ideal worst alternatives. This straight forward geometric system computation led to the increased popularity of the TOPSIS technique (Ding and Kamaruddin 2015). The popularity of the TOPSIS technique is quite evident by the number of its applications in different areas (Behzadian et al. 2012). It is obvious that while considering attributes such as material strengths, corrosion resistance, and toxic harm rate, the losses cannot be justified using the Euclidian distances alone. The losses caused may be in quadratic or in cubic or may be in some other form. In addition, instead of the Euclidean distances and the relative closeness measures, the loss for not choosing the best alternative can be understood easily. Hence, in the present work, instead of relative closeness measure, the total loss for not choosing the best alternative is suggested. As the actual losses are very difficult to measure, relative losses are fixed at one for the worst value of the attribute and zero for the best value of the alternative. After incorporating the weights, Eq. (27) can be used in the calculation of the losses. As the calculation of the losses is so simple and the results obtained are very close to the best solutions obtained by the other methods, the proposed method can be used as a simple and effective method for MADM problems.

Regarding the use of loss function approach, it is worth mentioning that the concept of loss function is being used by the statisticians well before the introduction of Taguchi's loss function (Ferguson 1967). Taguchi et al. (1989) applied the concept of quadratic loss function to quantify the quality loss. With the assumption that quality loss is zero when the quality characteristic is at target value and the maximum losses occurring at upper and lower specifications, the quality loss is approximated to follow a quadratic function. The total quality loss for $\boldsymbol{a}$ number of products with different individual measurements is given by:

Total loss $=K\left[a(\mu-\tau)^{2}+(a-1) \sigma^{2}\right]$

where $K$ is the constant of proportionality, $\mu$ is the mean of the measurements and $\sigma^{2}$ is the sample variance.

In the present study, the concept of loss function is introduced in MADM problems to make decision making simple so that the industrial personnel without much mathematical background can easily understand. Different loss functions with indices $n=1,2$ and 3 have been introduced in the analysis. The total loss of an alternative for not being the ideal best is derived as Eq. (27). It is quite obvious that the equations for Taguchi's total loss (28) and the total loss proposed in the present study (27) are entirely different. Maximum loss in the case of Taguchi's loss function is assumed to occur at the specification limits (upper specification and lower specification) and are estimated based on repair/failure costs. In the proposed method, the maximum loss is assumed to be one for any attribute belonging to the ideal worst alternative. With the introduction of attributes weights, the maximum loss is made equal to the weight of the attribute under consideration.

In addition, it can be observed from the literature that different MADM methods provide different solutions (order of preferences) for the same problem. Sometimes it may so happen that the last preference of one method becomes the first choice of some other method. For example, the robot selection problem involving three different robots (Rao 2007) using three popular MADM methods provided the following solutions:

GTMA: Robot 2 - Robot 1 - Robot 3

AHP: Robot 3 - Robot 1 - Robot 2

Modified TOPSIS: Robot 1 - Robot 3 - Robot 2 .

Naturally, the decision maker would be in a confusion in choosing the right decision. The concern for any decision maker in such a situation is to know the consequence of a wrong decision. The proposed loss function approach gives a convincing solution in terms of the total loss for each alternative.

\section{Proposed loss function approach with examples}

It is argued that considering the straight line-based distance measures as in TOPSIS may not be always appropriate because the losses incurred for not choosing the best would not be always linearly related to distances. To highlight this observation, loss function approach with different loss indices has been proposed. Different attributes may possess different indices, but for simplicity of explanation the proposed loss function approach has been employed to solve MADM problems with the following assumptions:

1. The index of loss function for each attribute can be assumed safely with prior knowledge about the attributes.

2. The loss function index is the same for all attributes of different alternatives.

The following are the steps involved in implementing the proposed loss function approach (LFA) of decision making.

Step 1 Obtain the data pertaining to all available alternatives. If any of the attributes in the data contain qualitative information, convert the qualitative data to quantitative data using an appropriate technique.

Step 2 The attributes are to be classified as non-beneficial or beneficial attributes based on the nature of the attributes. If a maximum value is desired for the attribute under consideration, it is taken as a beneficial attribute. If a 
minimum value is desired for an attribute, it is taken as non-beneficial attribute.

Step 3 Obtain the weight of each attribute using one of the existing methods. Because of the effectiveness and popularity, Analytical Hierarchy Process (AHP) method is preferred in obtaining the weights for each attribute.

Step 4 Assign a maximum loss for each attribute equal to the weight of the corresponding attribute.

Step 5 Decide upon the appropriate loss function to be used for each attribute. The loss functions proposed are linear or quadratic or cubic functions.

Step 6 Calculate the loss of choosing an alternative using Eq. (24) or (25) or (26). Arrange the alternatives in the ascending order of their loss values. This gives the order of preference for each alternative.

The following section illustrates some of the MADM problems using the proposed loss function approach. Initially, the problems are solved using the proposed loss function approach. The solutions obtained are then compared with those obtained from the literature using some of the MADM methods, namely AHP, TOPSIS, modified TOPSIS and GTMA.

\section{Machinability evaluation}

Rao (2007) solved the machinability evaluation problem using the data (Table 2) obtained from turning of various work materials by employing MADM techniques AHP, TOPSIS, modified TOPSIS and GTMA. Data of Table 2 are the results of experimentation conducted on ferrous and nonferrous alloys with high-speed machining tools (Bech 1963; Konig and Erinski 1983). The three attributes in the present problem are: 1-hour cutting speed (VC), specific cutting force (CF), and cutting power input (PI). A work material allowing very high cutting speeds is considered to possess high machinability characteristic. Hence, 1-hour cutting speed (VC) is a beneficial attribute. The remaining two attributes, namely specific cutting

Table 2 Objective data of the alternative alloys

\begin{tabular}{lccc}
\hline Work material & VC $(\mathrm{m} / \mathrm{min})$ & $\mathrm{CF}\left(\mathrm{N} / \mathrm{m}^{2}\right)$ & PI $(\mathrm{kW})$ \\
\hline W1 & 710 & 400 & 28 \\
W2 & 900 & 415 & 38 \\
W3 & 1630 & 440 & 59 \\
W4 & 1720 & 235 & 43 \\
W5 & 120 & 1150 & 8 \\
W6 & 160 & 1750 & 19 \\
\hline
\end{tabular}

W1 GK-A1Si10 Mg (aluminum-silicon die-cast alloy), W2 GKAlSi6Cu4 (aluminum-silicon die-cast alloy), W3 GK-AlMg5 (aluminum-magnesium die-cast alloy), W4 GK-MgA19Zn (magnesiumaluminum die-cast alloy), W5 GG26 (gray cast iron with lamellar graphite), W6 C35 (low-carbon steel) force $(\mathrm{CF})$ and cutting power input $(\mathrm{PI})$ are non-beneficial attributes.

The weight for each attribute has been considered from the study by Rao (2007) for comparison purpose. The weights are in fact obtained using the AHP method. The normalized weight for each attribute is given as: $W_{\mathrm{VC}}=0.7142, W_{\mathrm{CI}}=0.1429$, and $W_{\mathrm{PI}}=0.1429$.

\section{Solution using linear, quadratic and cubic loss functions}

The procedure to obtain the solution is explained stepwise:

Step 1 The information regarding all the alternatives is given in Table 2. The first column of Table 2 shows the different alternatives available (W1 to W6). There are three attributes, namely VC, CF and PI. The information on all attributes is quantitative. Hence, the process of converting the qualitative data to quantitative data is not required.

Step 2 The attribute VC is recognized as a beneficial attribute and the attributes $\mathrm{CF}$ and PI are non-beneficial attributes.

Step 3 There are three attributes in the present problem. The weight for each attribute is obtained using AHP process.

Weight of the first attribute $(\mathrm{VC}), w_{\mathrm{H}}^{1}=0.7142$.

Weight of the second attribute $(\mathrm{CF}), w_{\mathrm{S}}^{2}=0.1429$.

Weight of the third attribute (PI), $w_{\mathrm{S}}^{3}=0.1429$.

Step 4 Since the attribute VC is a beneficial attribute, the maximum loss occurs when the attribute takes on a minimum value. Similarly, the maximum losses occur when CF and PI are at their minimum values.

Step 5 The present problem is solved based on linear, quadratic and cubic loss functions.

Initially, consider linear loss function:

From Table 2, the pertinent information is obtained as follows:

$$
\begin{array}{lll}
x_{\max }^{1}=1720 ; & x_{\max }^{2}=1750 ; & x_{\max }^{3}=59 \\
x_{\text {min }}^{1}=120 ; & x_{\text {min }}^{2}=235 ; & x_{\text {min }}^{3}=8
\end{array}
$$

The losses for choosing different alternatives are now calculated using Eq. (24) and the respective losses are given below:

$$
L_{1}=0.5224, L_{2}=0.4671, L_{3}=0.2024, L_{4}=0.0981 \text {, }
$$
$L_{5}=0.8005$ and $L_{6}=0.8701$.

The solution obtained using the linear loss function can be given as:

$\mathrm{W} 4>\mathrm{W} 3>\mathrm{W} 2>\mathrm{W} 1>\mathrm{W} 5>\mathrm{W} 6$

Using quadratic loss function, the losses obtained for the different alternatives are given below:

$$
L_{1}=0.3038, L_{2}=0.2391, L_{3}=0.1473, L_{4}=0.0673 \text {, }
$$
$L_{5}=0.7$ and $L_{6}=0.8285$. 
Table 3 A comparison of the solutions for Machinability Evaluation problem

\begin{tabular}{|c|c|}
\hline Method & Solution \\
\hline AHP & $\mathrm{W} 4>\mathrm{W} 3>\mathrm{W} 2>\mathrm{W} 1>\mathrm{W} 5>\mathrm{W} 6$ \\
\hline TOPSIS & $\mathrm{W} 4>\mathrm{W} 3>\mathrm{W} 2>\mathrm{W} 1>\mathrm{W} 5>\mathrm{W} 6$ \\
\hline Modified TOPSIS & $\mathrm{W} 4>\mathrm{W} 3>\mathrm{W} 2>\mathrm{W} 1>\mathrm{W} 5>\mathrm{W} 6$ \\
\hline GTMA & $\mathrm{W} 4>\mathrm{W} 3>\mathrm{W} 2>\mathrm{W} 1>\mathrm{W} 5>\mathrm{W} 6$ \\
\hline LFA-1 & $\mathrm{W} 4>\mathrm{W} 3>\mathrm{W} 2>\mathrm{W} 1>\mathrm{W} 5>\mathrm{W} 6$ \\
\hline LFA-2 & $\mathrm{W} 4>\mathrm{W} 3>\mathrm{W} 2>\mathrm{W} 1>\mathrm{W} 5>\mathrm{W} 6$ \\
\hline LFA-3 & $\mathrm{W} 4>\mathrm{W} 3>\mathrm{W} 1>\mathrm{W} 2>\mathrm{W} 5>\mathrm{W} 6$ \\
\hline
\end{tabular}

Hence, the solution obtained using the quadratic loss is given below:

$\mathrm{W} 4>\mathrm{W} 3>\mathrm{W} 2>\mathrm{W} 1>\mathrm{W} 5>\mathrm{W} 6$

Finally, using the cubic loss function, the losses for choosing for different alternatives are given below:

$L_{1}=0.1885, L_{2}=0.2391, L_{3}=0.1473, L_{4}=0.0673$, $L_{5}=0.7663$ and $L_{6}=0.8285$.

The solution obtained using cubic loss function is given below:

$\mathrm{W} 4>\mathrm{W} 3>\mathrm{W} 1>\mathrm{W} 2>\mathrm{W} 5>\mathrm{W} 6$

A comparison of the solutions obtained using the proposed method with those reported in the literature (Rao 2007) for the same problem using AHP, TOPSIS, modified TOPSIS and GTMA methods is provided in Table 3.

From Table 3, it can be observed that all the methods proposed the alternative 4 (W4) as the best alternative. In addition, in the order of preference, all methods are in close agreement with each other except the LFA-3. The cubic loss function resulted in slightly different ranking.

\section{Machine group selection}

As a second problem for illustrating the proposed loss function approach, machine group selection problem from Wang et al. (2000) is considered. The objective data obtained for ten alternatives are given in Table 4 . The problem consists of four attributes, namely total purchasing cost (PC), total floor space (FS), total machine number $(\mathrm{MN})$ and productivity $(\mathrm{P})$. Out of four attributes under consideration, productivity is the only beneficial attribute, which needs to be maximized. All the remaining attributes (PC, FS and MN) are non-beneficial. The weights of the attributes are taken as $W_{\mathrm{PC}}=0.467, W_{\mathrm{FS}}=0.16$, $W_{\mathrm{MN}}=0.095$ and $W_{\mathrm{P}}=0.278$. These weights are taken from the study by Rao (2007) for comparison purpose.

Using the loss function approach with linear, quadratic and cubic loss functions, the machine group selection problem is solved. The resulting losses for all the alternatives under the above three loss functions are given in Table 5 .

Using the results of Table 5, the order of preference for the ten alternatives can be found. A comparison of the results obtained using loss function approach with the results of MADM methods available in the literature (Rao 2007) are shown in Table 6.

From the results of Table 6 , it can be observed that all the methods under consideration suggest the alternative 4 as the best choice. The first four alternatives suggested by AHP, TOPSIS, LFA-1, and LFA-2 are the same and also the last preference is given to alternative 2 by all these methods. Even all loss function approach-based methods suggest the alternative 2 as the last preference. GTMA prefers alternative 2 in comparison with the alternatives 8 , 9 and 10 which cannot be justified. From Table 4, it can be observed that the total purchasing cost (PC) is low for the alternatives 8,9 and 10 compared to the alternative 2 . The total purchase cost (PC) is maximum for alternative 2 and minimum for alternative 8 . In addition, the productivity $(\mathrm{P})$ of alternatives 8,9 and 10 are much superior compared to that of alternative 2. Moreover, the weights of $\mathrm{PC}$ and $\mathrm{P}$ are very high compared to the remaining attributes FS and MN. These facts show the superiority of the order of preference suggested by AHP, TOPSIS, LFA1, LFA-2 and LFA-3.
Table 4 Objective data for machine group selection problem

\begin{tabular}{lllll}
\hline Alternative & $\begin{array}{l}\text { Total purchasing } \\
\text { cost }(\mathrm{PC})(\$)\end{array}$ & $\begin{array}{l}\text { Total floor space } \\
(\mathrm{FS})\left(\mathrm{m}^{2}\right)\end{array}$ & $\begin{array}{l}\text { Total machine } \\
\text { number }(\mathrm{MN})\end{array}$ & $\begin{array}{l}\text { Productivity } \\
(\mathrm{P})(\mathrm{mm} / \mathrm{min})\end{array}$ \\
\hline 1 & 581,818 & 54.49 & 3 & 5500 \\
2 & 595,454 & 49.73 & 3 & 4500 \\
3 & 586,060 & 51.24 & 3 & 5000 \\
4 & 522,727 & 45.71 & 3 & 5800 \\
5 & 561,818 & 52.66 & 3 & 5200 \\
6 & 543,030 & 74.46 & 4 & 5600 \\
7 & 522,727 & 75.42 & 4 & 5800 \\
8 & 486,970 & 62.62 & 4 & 5600 \\
9 & 509,394 & 65.87 & 4 & 6400 \\
10 & 513,333 & 70.67 & 4 & 6000 \\
\hline
\end{tabular}


Table 5 Loss obtained using linear, quadratic and cubic functions

\begin{tabular}{llll}
\hline Alternative & Linear function & Quadratic function & Cubic function \\
\hline 1 & 0.5873 & 0.4333 & 0.3458 \\
2 & 0.7666 & 0.7479 & 0.7454 \\
3 & 0.6612 & 0.5461 & 0.4681 \\
4 & 0.2417 & 0.0785 & 0.0255 \\
5 & 0.5352 & 0.3420 & 0.2255 \\
6 & 0.6082 & 0.4188 & 0.3252 \\
7 & 0.4967 & 0.3335 & 0.2805 \\
8 & 0.3031 & 0.1961 & 0.1453 \\
9 & 0.3001 & 0.1886 & 0.1491 \\
10 & 0.4014 & 0.2478 & 0.1992 \\
\hline
\end{tabular}

Table 6 A comparison of the results of machine group selection problem

\begin{tabular}{ll}
\hline Method & Solution \\
\hline AHP & $4>9>8>10>5>1>7>3>6>2$ \\
TOPSIS & $4>9>8>10>7>5>1>6>3>2$ \\
Modified TOPSIS & $4>9>5>1>8>3>10>2>7>6$ \\
GTMA & $4>5>1>3>2>9>8>10>7>6$ \\
LFA-1 & $4>9>8>10>7>5>1>6>3>2$ \\
LFA-2 & $4>9>8>10>7>5>6>1>3>2$ \\
LFA-3 & $4>8>9>10>5>7>6>1>3>2$
\end{tabular}

\section{Material selection problem}

As a last example to illustrate the proposed loss function approach method, material selection problem of Manshadi et al. (2007) has been selected. Rao (2007) provides solutions to the same materials selection problem using GTMA, AHP, TOPSIS and modified TOPSIS methods. The number of materials under consideration is seven. Each material is characterized by seven important properties. They are toughness index (TI), yield strength (YS), Young's modulus (YM), density (D), thermal expansion (TE), thermal conductivity (TE) and specific heat (SH). The objective data of the seven materials are provided in Table 7.

Out of the seven attributes, the first three attributes TI, YS and YM are beneficial. Remaining four attributes, D, $\mathrm{TE}, \mathrm{TC}$ and $\mathrm{SH}$, are non-beneficial. To have comparison with the previous solutions, the weights are chosen to be same as that by Rao (2007). The weight for each attribute is as follows:

$$
\begin{aligned}
& W_{\mathrm{TI}}=0.24 ; \quad W_{\mathrm{YS}}=0.14 ; W_{\mathrm{YM}}=0.05 ; \quad W_{\mathrm{D}}=0.24 ; \\
& W_{\mathrm{TE}}=0.19 ; \quad W_{\mathrm{TC}}=0.05 \text { and } W_{\mathrm{SH}}=0.05
\end{aligned}
$$

Table 7 Objective data of material selection problem

\begin{tabular}{lcrlllll}
\hline Material & \multicolumn{7}{l}{ Material selection attributes } \\
\cline { 2 - 8 } & TI & YS & YM & D & TE & TC & SH \\
\hline 1 & 75.5 & 420 & 74.2 & 2.8 & 21.4 & 0.37 & 0.16 \\
2 & 95 & 91 & 70 & 2.68 & 22.1 & 0.33 & 0.16 \\
3 & 770 & 1365 & 189 & 7.9 & 16.9 & 0.04 & 0.08 \\
4 & 187 & 1120 & 210 & 7.9 & 14.4 & 0.03 & 0.08 \\
5 & 179 & 875 & 112 & 4.43 & 9.4 & 0.016 & 0.09 \\
6 & 239 & 1190 & 217 & 8.51 & 11.5 & 0.31 & 0.07 \\
7 & 273 & 200 & 112 & 8.53 & 19.9 & 0.29 & 0.06 \\
\hline
\end{tabular}

$T I$ toughness index, $Y S$ yield strength (MPa), YM Young's modulus (GPa), $D$ density $\left(\mathrm{g} / \mathrm{m}^{3}\right), T E$ thermal expansion $\left(10^{6} /{ }^{\circ} \mathrm{C}\right), T C$ thermal conductivity $\left(\mathrm{cal} / \mathrm{cm}^{2} / \mathrm{cm} /{ }^{\circ} \mathrm{C} / \mathrm{s}\right), S H$ specific heat $\left(\mathrm{cal} / \mathrm{g} /{ }^{\circ} \mathrm{C}\right)$

With the above weights and using the linear, quadratic and cubic loss functions, the total losses for each alternative have been calculated and provided in Table 8 .

Using the results of Table 8, the order of preference for the seven materials under consideration is obtained. A comparison of the solutions using other MADM methods and present loss function approach methods is provided in Table 9.

From Table 9, it can be observed that results obtained using AHP, TOPSIS and LFA-1 method are the same. Similarly, the solutions obtained using modified TOPSIS, GTMA and LFA-2 are the same. Except LFA-3, all the methods preferred materials 3 and 5 as the first and second choices. In addition, with slight differences, all methods choose the materials 3, 4, 5 and 6 in the first four places. The least preferred materials are 1,2 and 7. It is appropriate to judge the superiority of any method based on the first preferences given by the method, since the decision maker is always interested to choose the best. Judging a method based on the last preference may not be much useful and would be illogical. In the present comparison, all methods are found to suggest 3, 4, 5 and 6 as the materials which deserve much attention. Even though the real loss function behavior is not exactly known for many attributes, it can be appropriate to choose loss function with linear or quadratic nature based on the encouraging results obtained so far.

\section{Summary and conclusions}

In the present work, a simple-to-understand and easy-toconvince method based on loss function approach has been proposed. The method is based on assigning a maximum loss of one to an undesirable value of the attributes and zero loss to the most desirable value of the attributes in the available range of values of the attributes. The total loss for choosing an alternative has been calculated using an appropriate loss function. Based on the losses, the 
Table 8 Loss obtained using linear, quadratic and cubic functions

\begin{tabular}{llll}
\hline Material & Linear function (LFA-1) & Quadratic function (LFA-2) & Cubic function (LFA-3) \\
\hline 1: Al 2024-T6 & 0.7169 & 0.6739 & 0.6433 \\
2: Al 5052-0 & 0.7465 & 0.7338 & 0.7220 \\
3: SS 301-FH & 0.3493 & 0.2614 & 0.2104 \\
4: SS 310-3AH & 0.5653 & 0.4252 & 0.3491 \\
5: Ti-6Al-4 V & 0.4146 & 0.2750 & 0.2065 \\
6: Inconel 718 & 0.5504 & 0.4449 & 0.3926 \\
7: 70Cu-30Zn & 0.7999 & 0.6858 & 0.5985 \\
\hline
\end{tabular}

Table 9 A comparison of the results of material selection problem

\begin{tabular}{ll}
\hline Method & Solution \\
\hline AHP & $3>5>6>4>1>2>7$ \\
TOPSIS & $3>5>6>4>1>2>7$ \\
Modified TOPSIS & $3>5>6>4>7>1>2$ \\
GTMA & $3>5>4>6>7>1>2$ \\
LFA-1 & $3>5>6>4>1>2>7$ \\
LFA-2 & $3>5>4>6>1>7>2$ \\
LFA-3 & $5>3>4>6>7>1>2$ \\
\hline
\end{tabular}

alternatives are ranked in the ascending order of their losses. Linear, quadratic and cubic loss functions have been used to explain the methodology. In all these cases, the loss functions chosen are convex. The reason for choosing convex functions is that the losses are assumed to be small when the attribute is near the desired values and the losses are assumed to increase at higher rates when the deviations increase. The solutions obtained are in agreement with the solutions obtained using other MADM methods such as TOPSIS, AHP and modified TOPSIS. A comparison of the solutions obtained for three industrial problems using the present approach and other methods is made for illustration purpose. The solutions would be very convincing if an appropriate loss function was chosen. In general, linear and quadratic loss functions are observed to be sufficient.

Apart from its simplicity in understanding the concept of total loss, the present method avoids the process of normalization, a major step in many MADM methods. Since the method involves simple arithmetic, calculation of losses is also very easy and interpretation of the results based on losses makes the decision maker more comfortable.

Finally, it can be pointed out that the solutions obtained are highly dependent on the weights assigned to each attribute. In the present work, the weights obtained using AHP method have been employed in finding the solution. To find the best solutions, decision maker has to choose always the most appropriate and reliable method for finding the weights of the attributes.

Open Access This article is distributed under the terms of the Creative Commons Attribution 4.0 International License (http://creative commons.org/licenses/by/4.0/), which permits unrestricted use, distribution, and reproduction in any medium, provided you give appropriate credit to the original author(s) and the source, provide a link to the Creative Commons license, and indicate if changes were made.

\section{References}

Bana e Costa CA, Vansnick J (1997) A theoretical framework for measuring attractiveness by a categorical based evaluation technique (MACBATH). In: Climaco J (ed) Multicriteria Analysis. Springer Verlag, Berlin, pp 15-25

Bech HG (1963) Untersuchung derZerspanbarkeit von Leichtmetallegierungen. Dissertation, RWTH, Aachen

Behzadian M, Otaghsana SK, Yazdani M, Ignatius J (2012) A stateof-the-art survey of TOPSIS applications. Expert Syst Appl 39:13051-13069

Benayoun R, Roy B, Sussman N (1966) Manual de Reference du Programme Electre, Noe de Synthese et Formation, 25. Direction Scientifique SEMA, Paris

Brans JP, Mareschal B, Vincke Ph (1984) PROMITHEE: a new family of outranking methods in MCDM. Oper Res IFROS 84:477-490

Brauers WKM, Zavadskas EK (2006) The MOORA method and its applications to privatization in a transition economy. Contr Cybern 35:445-469

Brauers WKM, Zavadskas EK (2010) Project management by MULTIMOORA as an instrument for transition economies. Techno Econ Dev Econ 16:5-24

Charnes et al (1978) Measuring the efficiency of decision making units. Eur J Oper Res 2:429-444

Chen S, Hwang CL (1992) Fuzzy multiple attribute decision making-methods and applications. Springer-Verlag, Berlin

Dasarathy BV (1976) SMART: similarity measure anchored ranking technique for the analysis of multidimensional data analysis. IEEE Trans Syst Man Cybern SMC 6(10):708-711

Deng JL (1989) Introduction to gray system theory. J Gray Syst $1: 1-24$

Ding S, Kamaruddin S (2015) Assessment of distance-based multiattribute group decision-making methods from a maintenance perspective. J Ind Eng Int 11:73-85

Ferguson TS (1967) Mathematical statistics-a decision theoretic approach. Academic Press Inc., Newyork

Fontela E, Gabus A (1974) DEMATEL, Innovative methods, Report No. 2, Structural analysis of the world problematique, Battle Institute, Geneva Research Centre

Fontela E, Gabus A (1976) DEMATEL observer. Battle Institute, Geneva Research Centre

Hafezalkotob and Hafezalkotob (2016) Extended MULTIMOORA method based on Shannon entropy weight for material selection. J Ind Eng Int 12:1-13 
Hwang CL, Yoon KP (1981) Multiple attribute decision making: methods and applications. Lecture notes in Economics and Mathematical Systems, 186, Springer-Verlag, New York

Jacquet-Lagreze E, Siskos Y (1982) Assessing a set of additive utility functions for multicriteria decision making-the UTA method. Eur J Oper Res 10:151-164

Kackar NR (1985) Off-line quality control, parameter design and the Taguchi method. J Qual Tech 17:176-188

Keeney RL, Raffia H (1993) Decision with multiple objectives: preference and value trade off s. Cambridge University Press, New York

Konig W, Erinski D (1983) Machining and machinability of aluminium cast alloys. CIRP Ann 32:535-540

Manshadi BD, Mahmudi H, Abedian A, Mahmudi R (2007) A novel method for materials selection in mechanical design: combination nonlinear normalization and a modified digital logic method. Mater Des 28:8-15

McCrimmon KR (1968) Decision making among multiple-attribute alternatives: a survey and Consolidation approach. RAND Memorandum, RM_4823-ARPA

Pawlak Z, Slowinski R (1994) Rough set approach to multi-attribute decision analysis. Eur J Oper Res 72:443-459

Qin J, Liu X, Pedrycz W (2015) An extended VIKOR method based on prospect theory for multiple attribute decision making under interval type-2 fuzzy environment. Knowl Based Syst $86: 116-130$
Rao RV (2007) Decision making in the manufacturing environment. Springer-Verlag, London

Rao RV (2013) Decision making in the manufacturing environment, vol 2. Springer-Verlag, London

Saaty TL (1980) The analytic hierarchy process. McGraw Hill, New York

Taguchi G, Elsayed EA, Hsiang CT (1989) Quality engineering in production systems. McGraw-Hill book company, Newyork

Wang TY, Shaw CF, Chen YL (2000) Machine selection in flexible manufacturing cell: a fuzzy multiple attribute decision making approach. Int J Prod Res 38:2079-2097

Wang X, Yang F, Wei H, Zhang L (2015) A new ranking method based on TOPSIS and possibility theory for multiple attribute decision making problem. Optik 126:4852-4860

Yager RR (1988) On order weighted averaging aggregation operators in multicriteria decision making. IEEE Trans Syst Man Cyber 18:183-190

Yang L, Deuse J, Jiang P (2013) Multiple attribute decision making approach for an energy-efficient facility layout design. Int $\mathbf{J}$ Adv Manuf Technol 66:795-807

Zavadskas EK, Kaklanskas A, Turskis Z, Tomosaitiene J (2008) Selection of effective dwelling house walls by applying attributes values determined at intervals. J Civil Eng Manag 14:85-93

Zeleny M (1982) Multiple criteria decision making. McGraw-Hill, New York 